Revue de droit comparé du travail et de la sécurité sociale

3 | 2020

La Directive 2019/1158 du 20 juin 2019 concernant l'équilibre entre vie personnelle et vie privée des parents et des aidants

Modifications de diverses lois afin de faire face à la diversification des formes de travail

Yojiro Shibata

(2) OpenEdition

Journals

Édition électronique

URL : https://journals.openedition.org/rdctss/1033

DOI : $10.4000 /$ rdctss. 1033

ISSN : 2262-9815

Éditeur

Centre de droit comparé du travail et de la sécurité sociale

Édition imprimée

Date de publication : 1 novembre 2020

Pagination : 192-195

ISSN : 2117-4350

Référence électronique

Yojiro Shibata, « Modifications de diverses lois afın de faire face à la diversification des formes de travail », Revue de droit comparé du travail et de la sécurité sociale [En ligne], 3 | 2020, mis en ligne le 01 novembre 2021, consulté le 11 novembre 2021. URL : http://journals.openedition.org/rdctss/1033 DOI : https://doi.org/10.4000/rdctss.1033

\title{
(c)
}

Revue de droit comparé du travail et de la sécurité sociale est mise à disposition selon les termes de la Licence Creative Commons Attribution - Pas d'Utilisation Commerciale - Pas de Modification 4.0 International. 


\section{MODIFICATIONS DE DIVERSES LOIS AFIN DE FAIRE FACE À LA DIVERSIFICATION DES FORMES DE TRAVAIL'}

Du 20 janvier au 17 juin 2020, la 201e session de la Diète qui s'est tenue à Tokyo a été consacrée aux différentes mesures destinées à faire face à la propagation du coronavirus. Pourtant, plusieurs autres lois ont également été adoptées. Parmi elles, la loi modifiant diverses lois relatives au droit social comme l'assurance chômage (Loi n¹4 du 31 mars 2020) vise notamment à mettre en place un «filet de sécurité » en faveur de la protection des salariés cumulant plusieurs emplois (I), à réviser la prestation de congé parental (II), et à obliger les employeurs à faire des efforts dans la prise de mesures pour assurer le travail (pas forcément l'emploi) des employés âgés jusqu'à 70 ans (III).

\section{I - LA PROTECTION DES SALARIÉS CUMULANT PLUSIEURS EMPLOIS}

L'un des objectifs poursuivis par la « réforme pour mieux travailler »² était de promouvoir l'activité professionnelle complémentaire des salariés ${ }^{3}$. De fait, certaines entreprises encouragent leurs salariés à avoir une autre activité professionnelle et, effectivement, le nombre des salariés cumulant plusieurs emplois augmente. En revanche, il n'existe pas de " filet de sécurité " propre aux salariés cumulant plusieurs emplois, ce qui a conduit la loi n¹4 à réviser les lois sur l'assurance accidents du travail (A) et chômage (B).

\section{A - LA RÉVISION DE LA LOI SUR L'ASSURANCE ACCIDENTS DU TRAVAIL}

L'activité professionnelle complémentaire pose trois problèmes en ce qui concerne I'assurance accidents du travail. Tout d'abord, l'évaluation de la charge de travail soulève des difficultés. À titre d'exemple, un salarié travaillant 40 heures par semaine dans l'entreprise $(A)$, et 25 heures dans la (B), peut développer une maladie cérébrale ou cardio-vasculaire causée par le surmenage, qui sera considérée comme un « accident causé par la charge de travail chez plusieurs employeurs ». Or, cette maladie devrait théoriquement être qualifiée de maladie professionnelle. Il est donc probable que le chef du Bureau de l'inspection des normes du travail accepte la causalité adéquate entre le travail et la maladie cérébrale - ou cardio-vasculaire - lorsqu'un salarié travaille au-delà de 100 heures supplémentaires

1 Cet article a pu être écrit grâce aux subventions offertes par la Société Japonaise pour la Promotion des Sciences (JSPS - KAKANHI nº de subvention 17K03415).

2 «Réforme pour mieux travailler » (Hatarakikata Kaikaku) instaurée par la loi n71-2018. Voir E. Kasagi et H. Nagano, "Actualité juridique internationale du Japon », Revue de droit comparé du travail et de la sécurité sociale, 2019/1, p. 198.

3 En 2018, le ministère de la Santé, de l'Emploi et des Affaires sociales a modifié un article dans le "règlement intérieur type » (bon nombre de PME rédigent leur règlement intérieur en y faisant référence). Ce règlement interdisait jusqu'alors aux salariés d'avoir une activité professionnelle complémentaire, mais c'est désormais autorisé à la condition que cette activité secondaire ait préalablement été déclarée à l'employeur. 
sur un mois (la durée légale du travail au Japon étant de 40 heures hebdomadaires). Cependant, jusqu'à présent, l'assurance accidents du travail au Japon évaluait la causalité susmentionnée sur le lieu de travail où un accident - ou une maladie - survenait, sans tenir compte de la charge globale ou accumulée dans plusieurs lieux de travail. Par conséquent, la maladie évoquée dans l'exemple ci-dessus n'était pas considérée comme une maladie professionnelle puisqu'aucune des deux entreprises n'avait demandé au salarié de travailler plus que la durée légale autorisée par la loi sur les normes du travail. Avec cette réforme, l'administration tient compte de la charge globale effectuée dans plusieurs lieux de travail quand elle qualifie une maladie cérébrale, de maladie professionnelle.

Par ailleurs, s'ajoute à cela le problème du taux de cotisation accidents du travail, supportée seulement par l'employeur sous la forme d'un système de bonus-malus (loi sur la collecte des cotisations accidents du travail). En effet, la cotisation due au titre des accidents du travail est établie par chaque établissement en fonction de l'activité exercée, mais aussi du nombre d'accidents de travail dans cet établissement. Si l'accident de travail est causé par la charge de travail pour plusieurs employeurs, il n'influence ni le taux de cotisation de l'entreprise (A), ni celui de la (B).

Enfin, le montant des prestations soulève également des difficultés. Prenons un autre exemple : un salarié rémunéré 2000 € par mois dans l'entreprise (C), et $500 €$ dans la (D), est victime d'un accident de travail alors qu'il œuvrait dans l'entreprise (D), et se trouve dans l'incapacité provisoire de travailler pour les deux entreprises. Auparavant, le montant des prestations était calculé sur la seule base du salaire de l'entreprise (D) et il existait donc un grand écart entre perte de salaire et montant des prestations. La réforme de 2020 permet d'augmenter le montant des prestations de l'assurance accidents du travail, en additionnant les salaires de plusieurs entreprises lors de leur calcul.

\section{B - LA RÉVISION DE LA LOI SUR L'ASSURANCE CHÔMAGE}

Les salariés dont la durée de travail hebdomadaire ne dépasse pas 20 heures sont exclus de l'assurance chômage. Toutefois, en ce qui concerne les salariés cumulant plusieurs emplois, même si un salarié a travaillé plus de 20 heures par semaine au total - par exemple 15 heures dans l'entreprise (E) et 15 heures dans la (F) - il était exclu de l'assurance chômage dès lors que la durée de travail hebdomadaire ne dépassait pas 20 heures dans chacune des entreprises où il travaillait. Avec la réforme de 2020, les salariés cumulant plusieurs emplois - et âgés de plus de 65 ans $^{4}$ - sont ainsi éligibles à l'assurance chômage, sous réserve d'une déclaration au ministère de la Santé, de l'Emploi et des Affaires sociales, et à condition que la durée totale du travail hebdomadaire dans les deux (et pas plus) entreprises où ils travaillent dépasse 20 heures. En outre, lorsqu'ils quittent leur emploi dans l'une des deux entreprises mais continuent leur activité dans l'autre, ils perçoivent une indemnité versée en une seule fois en tant qu'« assuré âgé de 65 ans et plus ».

Enfin, pour que l'assuré soit bénéficiaire de l'indemnité chômage, il lui est demandé d'avoir travaillé sur une durée de 12 mois cumulés durant 2 ans avant la perte de son emploi. S'agissant de la durée d'affiliation, on compte un mois d'affiliation quand un salarié travaille plus de 11 jours par mois mais, depuis la réforme, cela s'applique également en faveur des assurés dont le nombre de jours mensuels de travail est insuffisant dès lors que le salarié a travaillé plus de 80 heures.

4 Le champ d'application se limite aux salariés âgés de plus de 65 ans car cette réforme est expérimentale. 


\section{II - LA RÉVISION DE LA PRESTATION DE CONGÉ PARENTAL}

La prestation de congé parental a été créée en 1994, dans le cadre des " prestations pour la continuité de l'emploi » de l'assurance chômage. L'une des particularités du système japonais réside en ce que la prestation de congé parental ne se situe pas dans le cadre des allocations familiales mais bien dans celui de l'assurance chômage. Cela s'explique par le fait que la réduction ou la perte de salaire consécutive à un congé parental rend difficile la " continuité de l'emploi » et que cette situation est considérée comme un risque social assimilé au chômage. La prestation de congé parental poursuit donc l'objectif de compenser une partie des salaires perdus suite à la prise d'un congé parental, de favoriser le retour à l'emploi, et finalement de stabiliser l'emploi. Depuis sa création, la durée de la prestation du congé parental a été prolongée et son champ d'application a été étendu aux salariés en CDD à la suite de différentes réformes. Entre autres, le montant de la prestation - qui s'élevait à l'origine à $25 \%$ du salaire avant le congé - s'établit aujourd'hui à $67 \%$ durant les premiers 180 jours (et $50 \%$ à partir du $181^{\mathrm{e}}$ jour).

Toutefois, cette prestation est davantage perçue comme un dispositif politique de lutte contre la dénatalité, plutôt que comme une mesure destinée à favoriser l'emploi continu. De plus, le nombre de bénéficiaires et la somme totale de la prestation ne cessent d'augmenter, tant et si bien que cette prestation tend à devenir l'une des prestations d'assurance chômage les plus importantes. Ainsi, certains critiquent le fait que la prestation de congé parental prenne le pas sur la prestation de base de l'assurance chômage, cette dernière portant pourtant sur l'objectif principal de l'assurance chômage : la prévention du chômage, la promotion du retour à l'emploi et la garantie de pouvoir vivre décemment pour les chômeurs.

A cet égard, l'objectif de la loi sur l'assurance chômage (art. $1^{\mathrm{er}}$ ) a été modifié, de manière à ce que la prestation de congé parental puisse bénéficier aux «salariés qui prennent un congé afin d'élever leurs enfants », et non plus aux salariés qui rencontrent des difficultés pour continuer à travailler. En outre, ses dispositions ont été transférées dans une nouvelle section de la loi. Il convient également de signaler le fait que sa gestion financière est distincte de celle des "prestations pour la continuité de l'emploi », et que sa propre cotisation $(0,4 \%$ partagée par moitié entre l'assuré et l'employeur) est fixée dans les cotisations chômage. En résumé, la prestation de congé parental s'est transformée en une prestation visant à " garantir les ressources » pendant le congé parental, tout en perdant son objectif initial : la « continuité de l'emploi » des salariés qui souhaitent prendre le congé parental.

\section{III - LES MESURES DESTINÉES À ASSURER LE TRAVAIL DES SALARIÉS ÂGÉS JUSQU'À 70 ANS}

À l'heure actuelle, la loi sur la stabilisation de l'emploi des travailleurs âgés oblige l'employeur à prendre l'une des trois mesures suivantes pour assurer l'emploi de ses salariés âgés jusqu'à 65 ans : le recul de l'âge de départ obligatoire à la retraite jusqu'à 65 ans; l'introduction d'un système d'emploi continu pour les salariés âgés jusqu'à 65 ans $^{5}$; la suppression de l'âge de départ obligatoire à la retraite.

Cette loi a été modifiée afin d'aménager l'environnement de travail pour les personnes âgées, de soutenir le dynamisme de la société, et de permettre aux personnes âgées

5 Le 2) diffère du 1) en ce que le contrat de travail d'un salarié âgé se termine à la retraite, et que ce salarié âgé en conclut un nouveau comprenant de nouvelles conditions de travail. 
qui souhaitent travailler de continuer à utiliser leurs compétences dans un contexte de vieillissement rapide de la population et de diminution de la population totale. La loi propose ainsi diverses options destinées à assurer le travail (pas obligatoirement l'emploi) des salariés âgés jusqu'à 70 ans.

Plus précisément, les employeurs qui fixent un âge de départ obligatoire à la retraite à plus de 65 ans et moins de 70 ans sont obligés de faire des efforts 6 pour prendre l'une des mesures suivantes:

1) le recul de l'âge de départ obligatoire à la retraite jusqu'à 70 ans ;

2) l'introduction d'un système d'emploi continu pour les employés âgés jusqu'à 70 ans ;

3) la suppression de l'âge de départ obligatoire à la retraite.

Néanmoins, les employeurs peuvent être dispensés des efforts susmentionnées et, à la condition d'un accord avec le syndicat (ou représentant) majoritaire et dans la mesure où leurs salariés concernés le souhaitent, alors prendre l'une des mesures suivantes:

4) la conclusion régulière d'un contrat visant à confier des tâches à une entreprise lancée par leurs anciens employés âgés de 65 ans au moins et de 70 ans au plus ;

5) la conclusion régulière d'un contrat par lequel les anciens employés âgés de 65 ans à 70 ans au plus exercent des activités rémunérées de contribution sociale, que celles-ci soient mises en œuvre par l'employeur ou par une personne morale confiée ou financée par l'employeur.

Les différences entre les mesures destinées aux personnes âgées jusqu'à 65 ans et jusqu'à 70 ans sont de deux ordres. En premier lieu, les mesures pour les personnes âgées de 70 ans au plus comprennent les points 4) et 5) qui ne visent aucunement l'emploi des personnes âgées. Le droit du travail ne s'appliquant pas par conséquent à ces situations, cela peut générer des difficultés, telles que celles relatives à la détermination du montant minimal de la rémunération du contrat ou encore du dédommagement d'un accident ou maladie survenue lors de l'exécution dudit contrat. En second lieu, l'employeur d'un système d'emploi continu jusqu'à 65 ans se limite à être l'employeur initial, sa société filiale ou affiliée, tandis que le système d'emploi continu jusqu'à 70 ans s'étend à une autre entreprise, en dehors de la société filiale ou affiliée. Cette extension rend obscure la responsabilité juridique concernant le système d'emploi continu.

Enfin, le taux du complément salarial pour les personnes âgées baisse. Prévu par la loi sur l'assurance chômage, ce complément est alloué aux salariés âgés dans l'hypothèse où leur salaire à partir de 60 ans baisserait de plus de $25 \%$. II vise à maintenir, voire à augmenter, la volonté de travailler des personnes âgées, ainsi que d'aider et d'encourager les employeurs à employer des salariés âgés ${ }^{7}$. Considérant que l'emploi des salariés âgés jusqu'à 65 ans est devenu usuel et queles employeurs sont désormais tenus de faire des efforts pour prendre des mesures nécessaires afin d'assurer le travail des salariés âgés jusqu'à 70 ans, le rôle de ce complément sera moindre. C'est la raison pour laquelle la loi n 14 prévoit la réduction de son taux maximal de $15 \%^{8}$ à $10 \%^{9}$ du salaire à partir de 60 ans en 2025.

6 L'obligation de faire des efforts n'a aucune force contraignante (par exemple peine pénale), même si l'employeur n'en fait pas. Pourtant, cette obligation de faire des efforts sera, plus souvent, renforcée jusqu'à devenir une véritable obligation quelques années plus tard dans le domaine du droit du travail.

7 Parce que l'employeur peut réduire le salaire des personnes âgées après 60 ans.

8 Dans l'hypothèse où leur salaire à partir de 60 ans baisserait de plus de $39 \%$ avant leurs 60 ans.

9 Dans l'hypothèse où leur salaire à partir de 60 ans baisserait de plus de $36 \%$ avant leurs 60 ans. 